\title{
TELEHEALTH IN AUDIOLOGY - THE NEED AND POTENTIAL TO REACH UNDERSERVED COMMUNITIES
}

\author{
De Wet Swanepoel \\ Jackie L. Clark \\ Dirk Koekemoer \\ James W. Hall III \\ Mark Krumm \\ Deborah V. Ferrari \\ Bradley McPherson \\ Bolajoko Olusanya \\ Maurice Mars \\ lêda Russo \\ Jose Barajas
}

\section{ABSTRACT}

Permanent hearing loss is a leading global health care burden, with 1 in 10 people affected to a mild or greater degree. A shortage of trained healthcare professionals and associated infrastructure and resource limitations mean that hearing health services are unavailable to the majority of the world population. Utilizing information and communication technology in hearing health care, or tele-audiology, combined with automation offer unique opportunities for improved clinical care, widespread access to services, and more cost-effective and sustainable hearing health care. Tele-audiology demonstrates significant potential in areas such as education and training of hearing health care professionals, paraprofessionals, parents, and adults with hearing disorders; screening for auditory disorders; diagnosis of hearing loss; and intervention services. Global connectivity is rapidly growing with increasingly widespread distribution into underserved communities where audiological services may be facilitated through telehealth models. Although many questions related to aspects such as quality control, licensure, jurisdictional responsibility, certification and reimbursement still need to be addressed; no alternative strategy can currently offer the same potential reach for impacting the global burden of hearing loss in the near and foreseeable future. 


\section{INTRODUCTION}

Spoken communication is central to human interaction and constitutes the basis for virtually all social, educational, and corporate relationships globally (Olusanya, Ruben \& Parving, 2006). The faculty which most fundamentally underlies the development and utilization of spoken communication is the auditory system. It is therefore not surprising that the effect of hearing loss is pervasive and far-reaching. In newborns and young infants hearing loss severely restricts or prevents the development of spoken language with concomitant effects on reading comprehension, cognitive development, socio-emotional functioning and ultimately academic achievement (Yoshinaga-Itano et al, 1998; Yoshinaga-Itano, 2004; Moeller et al, 2007).

Detection of hearing loss during the critical early developmental periods is essential to establishing appropriate remediation to prevent these direct and indirect effects of hearing loss, provided the necessary audiological services are available. In older individuals and adults hearing loss has a decisively negative impact on aspects such as social participation, emotional and behavioral well-being, and employment status. Hearing loss often leads to isolation and feelings of uncertainty, anger, anxiety and increased stress with rippling effects on families, significant others and the pursuit of quality interpersonal communication (Olusanya, Ruben \& Parving, 2006). All of these psychosocial dimensions are closely related to the basic well-being needed to achieve a sense of quality of life.

Fortunately intervention options for hearing loss have improved dramatically and, unlike many other chronic conditions, offer the possibility to limit the negative consequences and ensure significantly improved outcomes. Children with hearing loss who are enrolled in early hearing detection and intervention (EHDI) programs benefit from significantly altered developmental tracks, approximating those of normal hearing peers, as opposed to the persistent speech/language delays of their peers identified at a later age (Yoshinaga-Itano et al, 1998; Yoshinaga-Itano, 2004; Moeller et al, 2007). Aural rehabilitation, including amplification and subsequent counseling and intervention services for older children and adults are also characterized by marked improvements in outcomes, whether in developed or developing 
world contexts (Olusanya, 2004). Audiological services can provide early detection, diagnostic precision and uniquely personal hearing health care solutions that offer improved outcomes for all patients with hearing loss and related auditory disorders. Underpinning these benefits are integrated follow-up systems that efficiently transition patients from identification through diagnosis and onto intervention.

Unfortunately, the vast majority of persons with hearing loss globally are not identified early, are unable to access diagnostic services, and have no intervention options available to them (WHO, 2008a). The tremendous disparity between the virtual absence of global hearing health care and the proven benefits of these services raise important questions: What are the barriers to hearing health care and what may be done to improve access to hearing health care for persons with hearing loss? This paper addresses these issues by summarizing the need for increasing the global reach of hearing healthcare and surveying the potential scope and application of telehealth approaches in bringing audiological services to underserved regions of the world.

\section{GLOBAL BURDEN OF HEARING LOSS}

The World Health Organization estimates that hearing loss is the most prevalent disabling condition globally (WHO, 2008a). In 2005 the global prevalence of disabling (>40dB HL) hearing loss was estimated at 278 million, rising to 642 million, or almost $10 \%$ of the global population, when including mild hearing losses in the range of 26 to $40 \mathrm{~dB} \mathrm{HL}(\mathrm{WHO}, 2006 \mathrm{a})$. Permanent bilateral hearing loss in infants $(>40 \mathrm{~dB} \mathrm{HL})$ is estimated to affect approximately 798,000 newborns annually with more than $90 \%$ of these residing in developing countries (Olusanya \& Newton, 2007; Olusanya et al, 2009). Hearing loss in adults is even more prevalent, affecting one in every four individuals over the age of 45 years, with $27 \%$ of men and $24 \%$ of women in this age group presenting with hearing loss (Lopez et al, 2006; WHO, 2008a). 
Given its prevalence it is not surprising that hearing loss ranks as one of the leading contributors to the global burden of disease. Hearing loss ranks third on the global causes of years lived with disability (YLD) index and in high-income countries it ranks second (third in low- and middle-income countries; WHO, 2008a). The YLD index represents the loss of healthy life due to disability estimated using the years of life lived with the disability (Ali Hyder \& Morrow, 2006). When considering all health care conditions on the Disability Adjusted LifeYears (DALY) index, adult-onset hearing loss (ranked $15^{\text {th }}$ ) is one of only four non-fatal conditions among the 20 leading causes of global burden of disease (WHO, 2008a). The DALY index combines time lost due to disability with time lost due to death, that is, life that would have been expected had the disease not occurred (Ali Hyder \& Morrow, 2006). On this same index, hearing loss ranks $6^{\text {th }}$ in high-income countries and for women between 15 to 44 years of age, it ranks $10^{\text {th }}(\mathrm{WHO}, 2008 \mathrm{a})$. Future projections indicate hearing loss will be increasing in these rankings, estimated to be the $7^{\text {th }}$ leading cause of the global burden of disease in 2030, primarily due to a growing global population with increasingly long life expectancies (WHO, 2008a).

These projections may in fact still be underestimating the burden of global hearing loss, as was recently argued by Olusanya and Newton (2007). Current estimates only consider adultonset hearing loss and exclude permanent congenital and early-onset hearing loss (PCEHL) since this category is apparently accounted for by sequelae of other congenital conditions, infectious disease or injuries (Lopez et al, 2006). However, this is not sufficient to account for the burden of PCEHL. Most of the congenital and acquired causes were omitted in the estimates. At least $50 \%$ of causes are genetic and a significant percentage may be unknown whilst other major causes such as rubella, CMV, toxoplasmosis, mumps, herpes, neonatal jaundice and ototoxicity were also excluded (Olusanya \& Newton, 2007). Considering that annually an estimated 798,000 infants are born with or acquire permanent bilateral hearing loss in the first few weeks of life (more than 2000 daily), and global life expectancy exceeds 67 years, the life-long burden of global PCEHL may in fact exceed that of adult onset hearing loss (WHO, 2008b; Olusanya et al 2009; Swanepoel, Storbeck \& Friedland, 2009). Combining 
PCEHL and adult-onset hearing loss into a single category will result in a significantly larger contribution to the current global burden of disease than the current WHO estimates (Olusanya \& Newton, 2007).

Hearing loss is a serious disability which leads to significant social and economic burdens not only on individuals and their families but also on the resources of communities and countries (WHO, 2006a). The heaviest burden is evident in low- and middle-income countries where preventative, diagnostic and intervention services are often unavailable or unaffordable. In developing countries where illiteracy levels are very high and orality is central to life and culture, the effects of hearing loss will be even more accentuated and dramatic. This is made worse by the association between communication disorders, such as hearing loss, and poverty due to significantly higher unemployment rates that render affected individuals largely economically dependent (Olusanya, Ruben \& Parving, 2006). Hearing loss may be perpetuated in these poorer communities because of the exposure to more risks of hearing loss, such as unhygienic living conditions, disease outbreaks, lack of access to health care and poorer knowledge about prevention (Olusanya, Ruben \& Parving, 2006). Thus a cycle of hearing loss contributing to poverty and poverty contributing to hearing loss may be perpetuated.

\section{INADEQUACY OF GLOBAL HEARING HEALTH CARE SERVICES}

Nowhere is the irony of global inequality more striking than in hearing health care, with more than $80 \%$ of people with hearing loss residing in developing countries where services are either totally absent or very limited (WHO, 2006b; Fagan \& Jacobs, 2009). Despite being the most prevalent disabling condition globally and one of the major contributors to the global burden of disease, hearing loss has historically been ignored on global health care agendas. According to the WHO (2008a) it is "easily overlooked and underestimated" because it is not as "dramatic" as other health care conditions. It is therefore not surprising that hearing loss has been referred to as a silent epidemic (WHO, 2008a; Swanepoel, 2008). The World Health Organization estimates that fewer than 1 in every 40 people who could benefit from hearing 
aids actually receive this device (WHO, 2006b). This means that a simple intervention option such as provision of hearing aids is available to less than $2.5 \%$ of individuals who can benefit from them. Despite widespread universal newborn hearing screening programs in countries like the USA and UK (where more than $90 \%$ of newborns are screened) very little hearing screening, apart from small-scale pilot programs, is performed in the rest of the world (Olusanya et al, 2007; Theunissen \& Swanepoel, 2008; Swanepoel, Störbeck \& Friedland, 2009) .

Hearing health care surveys confirm that the paucity of services is in large part due to the limited numbers of available hearing health care professionals globally (Goulios \& Patuzzi, 2008; Fagan \& Jacobs, 2009). The average ratio of audiologists to the general population in developing countries reportedly varies between one for every half a million people to as high as one for every 6.25 million people. A recent survey of countries in sub-Saharan Africa indicated that many countries do not have any audiology or otolaryngology services (Fagan \& Jacobs, 2009). In developed countries the average ratio for audiologists to people was one to every 20,000 (Goulios \& Patuzzi, 2008). These shortages of hearing health care professionals are primarily due to a reported lack of government funding, professional and public awareness, and, most significantly, available training programs (Goulios \& Patuzzi, 2008). Only two African countries, for example, indicated having any training programs in audiology and many countries also indicated that they had no otolaryngology training programs (Fagan \& Jacobs, 2009). Emigration of trained staff, for economic reasons, to developed economies is another factor leading to acute shortages of hearing health care professionals (McPherson, 2008). In addition, there is an unequal distribution of existing hearing health care providers who are primarily situated in metropolitan areas which often leaves vast territories underserved.

However, it is not only in developing countries where hearing health care services are inadequate. Even in developed countries like the USA, the demand or need for audiological services is significantly greater than the current capacity of professionals providing these 
services. Margolis \& Morgan (2008) recently compared the estimated number of audiograms required annually in the USA with the capacity of current professionals to provide these tests. According to these estimations, in the year 2000 there was an annual shortfall of 8 million audiograms and this was projected to increase to 15 million by 2050 . This may be one of the factors contributing to the poor penetration of hearing aids in a country like the USA, where only $22 \%$ of individuals who could benefit from a hearing aid actually receive one. It is interesting that despite the increased awareness of noise in the community, licensure of hearing aid providers in many states, and the advent of the clinical audiological doctorate, this figure has seen less than 5\% growth in the past three decades (Kochkin, 2005).

It is a global health care dilemma that many people with hearing loss are not able to access the services they need. This divide between services and patients results from multiple factors including the lack of hearing health care professionals, poor public and professional awareness, limited resources, geographical barriers such as distance and difficult or remote terrains, and natural barriers such as severe weather. These barriers are not limited to developing countries and can occur in high-income countries where pockets of underserved people reside, for example remote rural regions (i.e. parts of Australia and Alaska) or even inner-city communities. Globally, the majority of children and adults with hearing loss are isolated from the very services which may improve hearing and communication and reduce the potential negative effects of hearing loss on social interaction, education and vocational opportunity.

Current global health care efforts are clearly inadequate for reaching the vast majority of people with hearing loss. Addressing this insufficiency will require comprehensive, multipronged and contextually-responsive solutions that consider the political, infrastructure and resource realities of countries and regions (Fagan \& Jacobs, 2009). Current approaches for expanding hearing health care services must be reviewed critically and new complimentary means of bringing service to people, such as telehealth, should be investigated as a matter of high priority. 


\section{TELEHEALTH - A PROMISING PROSPECT FOR HEARING HEALTH CARE}

Telehealth offers unique opportunities for providing access to hearing health care services to underserved populations worldwide. The term telehealth refers to the utilization of information and communication technology in health care. Alternate terminology that has been used to describe the field includes telemedicine, online health, and e-health. Telehealth literally means "health care at a distance" (Wootton et al, 2009). More recently the specific field or specialty to which telehealth is applied has been preceded by the prefix "tele", e.g. tele-audiology, which refers to the application of telehealth to the practice of audiology. Telehealth can be employed in a synchronous, real-time manner (e.g. an assessment via interactive videoconferencing) or in an asynchronous, store-and-forward manner (e.g. digital picture emailed to health care provider), or a hybrid model encompassing synchronous and asynchronous aspects can be used (Krumm, 2007).

Utilization of information and communication technology in health care is important for improving clinical care and public health and as such, may provide a cost-effective and sustainable means of providing much needed audiological services to those populations identified as having restricted or limited access. The possible benefits are far reaching and apart from facilitating medical education, administration and research, telehealth may improve health care access, quality of service delivery, effectiveness and efficiency of health care, and ameliorate the inequitable distribution of health professionals globally (Wootton et al, 2009). Internet connectivity and technology is providing a bridge between patients and health care providers who may otherwise be separated by distance, location, geographical and weather barriers as well as economic barriers. This divide may be bridged not only between patients and health care providers but also improve access for isolated health care providers to resources like training, professional interaction and mentoring.

The rapid improvement and distribution of internet connectivity is providing an increasing opportunity for implementation of telehealth on a global scale. Connectivity around the world 
has grown exponentially with one in every 4 people worldwide having access to the internet in 2008. The majority of those without access are from China, India and Africa. However internet user growth in these regions is currently experiencing unprecedented growth, having exceeded $1000 \%$ over the past 8 years (Internet World Stats, 2009). Although internet penetration in a region like Africa is still only $5.6 \%$ and bandwidth costs are exceedingly expensive the continued development in connectivity technology is creating opportunities that were not previously possible (Internet World Stats, 2009). The large-scale roll-out of cellular networks across Africa is opening doors to telehealth in the most remote and underserved areas. Concerted efforts from governments in partnership with private corporations must however lead the way in these regions to expand connectivity and reduce bandwidth costs to allow for feasible telehealth applications.

Telehealth applications reveal promising results in a variety of health care fields including radiology, pathology, dermatology, otology, psychiatry and pediatrics. Applications have included such simple uses as email for sending an x-ray image or digital pictures of skin conditions to more complex use of videoconferencing for real-time patient assessments and desktop application sharing on computers to conduct remote clinical procedures such as intraoperative monitoring (Desai, 2009; Smith et al, 2008; Wynchank \& Fortuin, 2008; Shapiro et al, 2008; Kokesh et al, 2008; Adler, Yu \& Datta, 2009; Rao \& Lombardi, 2009). Telehealth is a valuable tool for education and training as illustrated by the reported success of videoconferenced tele-education in remote areas of South Africa and Brazil and telementoring for continuous reinforcement of surgical training and guided surgery in isolated areas of India (Mars, 2008; Melo, 2008; Mishra, Pradeep \& Mishra, 2009). The value of telehealth is not only limited to remote or isolated communities but the principles may also be employed to improve and streamline current practices by employing automation, integration and coordination of processes. 


\section{POTENTIAL SCOPE OF TELE-AUDIOLOGY}

In view of the potential impact of telehealth in hearing health care and its current benefit to many other areas of medical practice, it is surprising that the field of tele-audiology has been slow to develop. Early tele-audiology studies, incorporating remote programming of analog digital hearing aids, video otoscopy and otoacoustic emissions, began at least a decade ago (Birkmire-Peters, Peters and Whitaker, 1999; Schmiedge, 1997). However to date, only a limited number of studies, many of which are only pilot projects, have investigated the possible applications and validity of tele-audiology (Krumm, 2007). Initial reports are, however, very positive and the foreseeable impact can be far-reaching in all areas of audiological practice and education in underserved communities, as summarized in Table 1.

\section{Education and training}

The use of information and communication technology to facilitate education and training opportunities for audiologists, hearing health care paraprofessionals, and parents of children with hearing loss or even adults with hearing loss or auditory disorders, has already received some interest. Live workshops provided through videoconference facilities and interactive online training modules may all facilitate continued professional development and training for paraprofessionals or community health care workers on topics such as primary ear and hearing health care as outlined in recent WHO training manuals (WHO 2006a). For example, in Brazil interactive videoconferencing has proved to be an effective tool for training community health care workers regarding hearing health care in children (Melo, 2008). Also in this country, characterized by its vast distances, a telehealth training mechanism has been introduced for audiological support in remote clinics via online interactive on demand training and provision of second opinions (Krumm \& Ferrari, 2008). Professional development may be fostered by telementoring programs in specific areas of audiological practice such as pediatric auditory evoked potential assessments, newborn hearing screening programs, fitting and verification of hearing aids, assessment of difficult to test populations, and management of balance disorders. 
Table 1. Scope of application possibilities for telehealth in audiology

*usually involves a paraprofessional or trained volunteer to facilitate the telemedicine setup at the remote location whilst the health care provider (audiologist) is present remotely via interactive videoconferencing.

** usually involves a paraprofessional or trained volunteer to facilitate the telemedicine setup at the remote location whilst the health care provider (audiologist) is not present or available in real-time via interactive videoconferencing.

\begin{tabular}{|c|c|c|}
\hline \multirow{2}{*}{$\begin{array}{c}\text { FIELD OF } \\
\text { APPLICATION }\end{array}$} & \multicolumn{2}{|c|}{ SCOPE OF TELEHEALTH APPLICATIONS } \\
\hline & Synchronous* & Asynchronous** \\
\hline $\begin{array}{l}\text { EDUCATION/TRAINING } \\
\text { - Health care providers } \\
\text { - Paraprofesionals } \\
\text { - Parents }\end{array}$ & $\begin{array}{l}\text { - Real-time interactive videoconference } \\
\text { presentations } \\
\text { - Telementoring and guidance during } \\
\text { assessments or procedures } \\
\text { - Discussing difficult results/cases with } \\
\text { experienced clinicians }\end{array}$ & $\begin{array}{l}\text { - Interactive online training modules } \\
\text { - Posting questions via email or online } \\
\text { forums } \\
\text { - Requesting } 2^{\text {nd }} \text { opinions from experienced } \\
\text { clinicians }\end{array}$ \\
\hline $\begin{array}{l}\text { SCREENING } \\
\text { - Newborn Hearing } \\
\text { Screening } \\
\text { - School-entry hearing } \\
\text { screening } \\
\text { - Adult hearing screening } \\
\text { (i.e. occupational health) } \\
\text { - Vestibular screening }\end{array}$ & $\begin{array}{l}\text { - Real-time screening directed via interactive } \\
\text { videoconferencing and application sharing } \\
\text { - Quality control of screening via interactive } \\
\text { videoconferencing }\end{array}$ & $\begin{array}{l}\text { - Automated OAE/ABR screening } \\
\text { - Automated audiometry screening } \\
\text { - Internet-based hearing tests may be } \\
\text { valuable screening options }\end{array}$ \\
\hline $\begin{array}{l}\text { DIAGNOSIS } \\
\text { - Case History } \\
\text { - Otoscopy } \\
\text { - Immittance } \\
\text { - Otoacoustic Emissions } \\
\text { (OAE) } \\
\text { - Auditory Evoked Potentials } \\
\text { (AEP) } \\
\text { - Audiometry (pure tone \& } \\
\text { speech) } \\
\text { - Vestibular assessment } \\
\text { - Intra-operative monitoring }\end{array}$ & $\begin{array}{l}\text { - Case history via interactive } \\
\text { videoconferencing } \\
\text { - Video-otoscopy via interactive } \\
\text { videoconferencing and application sharing } \\
\text { directed by audiologist } \\
\text { - Immittance, OAE, AEPs via interactive } \\
\text { videoconferencing and application sharing. } \\
\text { - Placement of probe/electrodes etc guided } \\
\text { by audiologist and testing conducted via } \\
\text { application sharing } \\
\text { - PC-based audiometers facilitate remote } \\
\text { testing via interactive videoconferencing and } \\
\text { application sharing }\end{array}$ & $\begin{array}{l}\text { - A case-history can be taken electronically } \\
\text { (store-and-forward or electronic patient } \\
\text { file) } \\
\text { - Video-otoscopy (store-and-forward or } \\
\text { electronic patient file) } \\
\text { - Automated test sequences of immittance } \\
\text { and OAE completed beforehand and } \\
\text { emailed (store-and-forward or electronic } \\
\text { patient file) } \\
\text { - Automated audiometry (store-and-forward } \\
\text { or electronic patient file) }\end{array}$ \\
\hline $\begin{array}{l}\text { INTERVENTION } \\
\text { - Counseling } \\
\text { - Ear canal management } \\
\text { - Hearing aid selection, } \\
\text { fitting \& verification } \\
\text { - Cochlear implant mapping } \\
\text { - Intervention }\end{array}$ & $\begin{array}{l}\text { - Counselling and troubleshooting conducted } \\
\text { via interactive videoconferencing } \\
\text { - Ear canal management guided remotely by } \\
\text { audiologist via videoconferencing } \\
\text { - Hearing aids fitting guided and programmed } \\
\text { via interactive videoconferencing and } \\
\text { application sharing } \\
\text { - Verification of hearing aid via application } \\
\text { sharing and interactive videoconferencing } \\
\text { - Cochlear implant activation and mapping via } \\
\text { application sharing and interactive } \\
\text { videoconferencing } \\
\text { - Follow-up sessions via interactive } \\
\text { videoconferencing } \\
\text { - Home-based early intervention services via } \\
\text { interactive videoconferencing }\end{array}$ & $\begin{array}{l}\text { - Hearing aids may be pre-selected and } \\
\text { pre-programmed based on audiological } \\
\text { results } \\
\text { - Counselling sessions via interactive } \\
\text { videoconferencing may be preceded by } \\
\text { questions and complaints emailed } \\
\text { - Internet-based audiological counselling } \\
\text { programs } \\
\text { - Internet-based audiological treatment } \\
\text { programs (i.e. tinnitus) } \\
\text { - Internet-based auditory training programs } \\
\text { - Home-based intervention for infants may } \\
\text { be provided by recorded play sessions at } \\
\text { home sent through to interventionist for } \\
\text { evaluation }\end{array}$ \\
\hline
\end{tabular}

\section{Screening}

Audiological screening utilizing synchronous telehealth technology has been reported as comparable to face-to-face testing in school-aged children using otoscopy, immittance and pure tone audiometry and in newborns using distortion product otoacoustic emission (DPOAE) 
screening (Lancaster et al, 2008; Krumm et al, 2008). DPOAE screening demonstrated equivalent DPOAE response amplitudes (within test-retest reliability limits) for face-to-face versus remote testing, otoscopy and tympanometry were identical between conditions, and 188 of 193 pure tone frequencies screened corresponded in terms of screen result (Lancaster et al, 2008; Krumm et al, 2008). Synchronous screening usually utilizes trained assistants at remote locations to prepare patients for testing (i.e. placing earphones and probes) under the guidance and monitoring of the remote audiologist through interactive video. Actual testing is performed through application sharing software which allows the audiologist to remotely operate the equipment on-site. Since assistants (i.e. volunteers or paid screeners) are often used in screening programs like newborn hearing screening, telehealth may be utilized for the remote training of screening personnel (volunteers or paraprofessionals) and monitoring of testing procedures to ensure quality control of programs. Using remote computing applications, the software used by assistants to screen patients' hearing at one site can be seen on the computer display of the supervising audiologist located elsewhere. Consequently, using a telephone or interactive video, the supervising audiologist can verbally instruct assistants about screening procedures in real-time while watching their technique and patients' responses.

Asynchronous automated protocols may also prove useful for audiometric screening in adult populations in occupational health settings or internet-based hearing screening. With the asynchronous automated protocol, large scale hearing screening can be accomplished with a local facilitator tasked with patient scheduling, taking and recording relevant case history, initiating and monitoring equipment with automated protocols, and forwarding all pertinent information especially when the decision support software in the automated programme indicates that referral is necessary. Alternately, self hearing screening services may be available over the telephone or Internet as recently reported for screening adults and adolescents in the Netherlands (Smits, Kapteyn \& Houtgast, 2004; Smits, Merkus \& Houtgast, 2006). The speech-in-noise screening test uses triple digit stimuli presented in an adaptive paradigm and can be done quickly and with results comparable to other speech in noise tests 
(Smits, Kapteyn \& Houtgast, 2004; Smits, Merkus \& Houtgast, 2006). Obviously, the greatest benefit of this technique is accessibility since hearing screening may be only a phone call away.

\section{Diagnostic testing}

The diagnostic applications of tele-audiology span the entire test battery of commonly-used audiological procedures. A live case history may be taken with the use of a synchronous interactive videoconferencing setup but may be more efficiently completed through an electronic case history (e.g., completing an online form, by email, etc) ahead of time, in an asynchronous manner. In this way the audiologist can review the information before a test is conducted or with a review of test findings. Video-otoscopy and immittance, for example, have been proven to be reliable techniques for use in both synchronous and asynchronous telehealth models (Smith et al, 2008; Kokesh et al, 2008). Until further validation, diagnostic OAEs may best be performed synchronously with interactive video to monitor probe placement. This should preferably also be performed whilst continuously monitoring environmental noise levels at the remote testing site to ensure reliable recordings (Elangovan, 2005). Auditory evoked potentials can be used synchronously with interactive video, whereby the audiologist may monitor and direct the paraprofessional on aspects such as electrode and transducer placement before taking control of the software remotely through application sharing for assessments of auditory functioning or intraoperative monitoring (Towers et al, 2005). Vestibular assessments using electronystagmography or videonystagmography can be used within similar models (Yates \& Campbell, 2005).

Several options are available for conducting diagnostic pure tone and speech audiometry within the context of telehealth (Choi et al, 2007; Givens \& Elangovan, 2003; Krumm, Ribera, \& Klich, 2007; Ribera, 2005). Internet-based hearing tests have been proposed as a means of hearing testing although there are serious practical concerns such as calibration accuracy and lack of control on environmental noise levels (Bexelius et al, 2008). This application although controversial, may well serve a purpose of increasing public awareness and as a preliminary 
screening for hearing loss. Computer-based audiometers can be used more reliably from remote locations with application sharing and interactive video for synchronous testing, but the influence of environmental noise remains a significant concern (Elangovan, 2005). The development of new telehealth compliant audiometers may address these concerns by providing double attenuation (i.e. insert and circum-aural earphones), monitoring environmental noise continually through an external microphone, and including active noise cancellation features. An asynchronous application of diagnostic audiometry may be found in the utilization of validated automated audiometry protocols included in PC-based and telehealth compliant audiometers (e.g. Margolis et al, 2007; Margolis \& Morgan, 2008). The combination of tele-audiology and automated audiometry can be a powerful way of providing time and resource efficient audiometric evaluations, especially in regions where audiological services are limited or unavailable.

\section{Intervention services}

Reliable diagnostic applications provide the basis for timely and appropriate intervention services varying from referrals for medical treatment or support services to the selection and institution of audiological interventions. Complications of middle-ear pathology, such as otitis media, cholesteatoma and mastoiditis, which are not uncommon in developing countries (Akinpelu et al, 2008), may result in permanent hearing loss and even death if not identified

and treated early. Diagnostic hearing tests may provide the first detection of such cases and allow for timely medical referrals to be made. The provision of amplification to those with hearing loss in isolated or remote areas may be uniquely facilitated by telehealth applications for several reasons. Many of the newer thin tube-open ear hearing aid fit designs utilize stabilizers or disposable domes, thus negating the heavy demand for custom earmolds so that an instant fit on location could be attained directly after diagnosis in some cases. For those patients for whom custom earmolds are required, earmold impressions may be taken by trained facilitators under guidance from a remote audiologist through interactive video. Furthermore the programming of digital hearing aids is primarily based on computer software that can be adjusted remotely through application sharing. Fitting and verification of hearing 
aids have been successfully conducted through these same telehealth mechanisms (Wesendahl, 2003; Ferrari, 2006; Ferrari \& Bernardez-Braga, 2009). With the ease of remote hearing aid programming, it is reasonable to expect that cochlear implant mapping is also a desirable application. Persons with cochlear implants require regular mapping sessions and these have been conducted from remote locations through application sharing and videoconferencing without incident and with no significant differences between face-to-face and remote maps, and similar recorded threshold-neural response imaging values (Ramos et al, 2009).

Once testing and intervention plans have been determined, counseling and troubleshooting can also be facilitated through interactive videoconferencing. Options include internet-based counseling or treatment programs for which initial reports are demonstrating significant benefit for new hearing aid users and tinnitus patients (Laplante-Lévesque, Pichora-Fuller \& Gagné, 2006; Kaldo et al, 2008). Intervention for children with hearing loss that is often familycentered and home-based may also be provided through interactive videoconferencing equipment. Simple videoconferencing facilities utilizing commercially available software programs and webcams can provide affordable means of conducting live sessions remotely. In addition, web sites can be used in developing countries to provide training and support for services such as Early Hearing and Detection Intervention programs or support groups for individuals affected by hearing loss

\section{TELE-AUDIOLOGY - A NEW ERA}

The potential applications and possible impact of tele-audiology are significant. Underserved regions, such as Africa, may incorporate telehealth as a way to provide services through the volunteer efforts of audiologists from other world regions. Tele-Audiology Network (www.teleaudiology.org), a recently formed non-governmental / non-profit organization, is piloting this concept as a way to provide services to remote areas in developing countries where audiological services are unavailable. In countries where audiology is well established, tele-audiology may also be employed to reach isolated and remote communities (e.g. in 
Alaska, Appalachia, Canada etc) and to improve the efficiency of current services (e.g. employing internet-based counseling or treatment programs).

However, as a field in its infancy, many questions must be addressed within the new framework of providing audiological services remotely. Validation studies on the application possibilities and mechanisms of tele-audiology are required to ensure the potential benefits are comparable to or better than the current service delivery standards. The possibility of remote testing, which can cross state and national borders, poses its own unique set of questions related to licensure, jurisdictional responsibility, certification, reimbursement and quality control. National and international professional bodies and organizations must proactively develop standards, policies and protocols for every aspect of tele-audiology. Currently, organizations like the American Speech and Hearing Association and the American Academy of Audiology (ASHA, 2005; AAA, 2008) offer some basic guidelines and benchmarks but these are lacking in an international perspective necessary to govern the global scope of tele-audiology. In a response to the growing need, the International Society of Audiology is investigating the development of international guidelines and standards for tele-audiology and using it as a means of remote training in hearing healthcare. It is to be hoped that all stakeholders participate in this initiative to guide the field of tele-audiology as it grows to maturity.

\section{CONCLUSION}

The impact of telehealth is increasingly evident in several areas of health care and its scope is continually broadening with advances in technology and internet connectivity. The utilization of telehealth in direct patient care is widely reported and it also has an important role in training local health professionals and paraprofessionals in developing world regions (Wootton, 2009). The application of telehealth to hearing health care is an exciting and emerging field with a broad scope of application possibilities including training/education, screening, diagnosis and intervention. These services are not bound by distance or location and can bridge the gap between patients isolated from the audiological services they require. 
Although much work remains to be done in terms of validation studies and development of international guidelines, the promise of hearing health care services to those unable to access them has strong potential for realization through tele-audiology. Although not the answer to all challenges related to global hearing loss, there is no alternative strategy that can offer the same positive impact on the current hearing loss burden in the near and foreseeable future. It is therefore time to embrace and harness the potential benefits that improved connectivity and technology may afford and extend audiological services to all who could benefit from them.

\section{REFERENCES}

Adler J., Yu C. \& Datta M. 2009. The changing face of radiology: from local practice to global network. Med J Aust, 190, 20-23.

Akinpelu O.V., Amusa Y.B., Komolafe E.O., Adeolu A.A., Oladele A.O. \& Ameye S.A. 2008. Challenges in management of chronic suppurative otitis media in a developing country. $J$ Laryngol Otol,122, 16-20.

Ali Hyder A. \& Morrow R.H. 2006. Measures of health and disease in populations. In: M.H Merson, R.E. Black \& A.J. Mills (eds.) International Public Health. Sudbury: Jones \& Bartlett Publishers, pp. 1-42.

American Academy of Audiology 2008. The use of telehealth/telemedicine to provide audiology services. http://www.audiology.org/advocacy/publicpolicyresolutions/Documents/TelehealthResolution20 0806.pdf (Accessed 21 May 2009)

American Speech-Language-Hearing Association 2005. Audiologists Providing Clinical Services via Telepractice: Technical Report [Technical Report]. Available from www.asha.org/policy. (Accessed 21 May 2009) 
Bexelius C., Honeth L., Ekman A., Eriksson M., Sandin S., Bagger-Sjöbäck D. \& Litton J.E. 2008. Evaluation of an internet-based hearing test--comparison with established methods for detection of hearing loss. J Med Internet Res, 10, e32.

Birkmire-Peters D.P., Peters L.J. \& Whitaker L.A. 1999. A usability evaluation for telemedicine medical equipment: a case study. Telemed J, 5, 209-212.

Choi J., Lee H., Park C., Oh S. \& Park K. 2007. PC-Based Tele-Audiometry. Telemed J E Health, 13, 501-508.

Desai S. 2009. Telepathology and telecytology in developing countries. In: R. Wootton, N.G. Patil, R.E. Scott \& K. Ho (eds.) Telehealth in the developing world. London: Royal Society of Medicine Press Ltd, pp. 149-155.

Elangovan S. 2005. Telehearing and the Internet. Semin Hear, 26, 19-25.

Fagan J.J. \& Jacobs M. 2009. Survey of ENT services in Africa: Need for a comprehensive intervention. Global Health Action, DOI: 10.3402/gha.v2i0.1932

Ferrari D.V. 2006. Remote programming and verification as a mean to improve quality of hearing aid fitting. In: A.N. Rasmussen, T. Paulsen, T. Andersen \& C.B. Larsen (org.) Hearing aid fitting. Proceedings of the 21st Danavox Symposium Centertryk, pp. 531-544.

Ferrari D.V. \& Bernardez-Braga G.R. 2009. Remote probe microphone measurement to verify hearing aid performance. J Telemed Telecare, 15, 122-124.

Givens G. \& Elangovan S. 2003. Internet application to tele-audiology-"nothin' but net." Am J Audiol, 12, 50-65. 
Goulios H. \& Patuzzi R.B. 2008. Audiology education and practice from an international perspective. Int J Audiol, 47, 647-664.

Internet World Stats 2009. Internet usage statistics.

http://www.internetworldstats.com/stats.htm (Accessed19 May 2009).

Kaldo V., Levin S., Widarsson J., Buhrman M., Larsen H.C. \& Andersson G. 2008. Internet versus group cognitive-behavioral treatment of distress associated with tinnitus: a randomized controlled trial. Behav Ther, 39, 348-359.

Kochkin S. 2005. MarkeTrak VII: Hearing loss population tops 31 million people. Hear Rev, 12(7), 16-29.

Kokesh J., Ferguson A.S., Patricoski C., Koller K., Zwack G., Provost E. \& Holck P. 2008. Digital images for postsurgical follow-up of tympanostomy tubes in remote Alaska. Otolaryngol Head Neck Surg, 139, 87-93.

Koopman J., Davey E., Thomas N., Wittkop T. \& Verschuure, H. 2008. How should hearing screening tests be offered? Int J Audiol, 47, 230-237.

Krumm M. 2007. Audiology telemedicine. J Telemed Telecare, 13, 224-229.

Krumm M., Ribera J., Klich R. 2007. Providing basic hearing tests using remote computing technology. J Telemed Telecare, 13, 406-410.

Krumm M., Huffman T., Dick K. \& Klich R. 2008. Telemedicine for audiology screening of infants. J Telemed Telecare.14, 102-104. 
Krumm M. \& Ferrari D.V. 2008. Contemporary telehealth and telemedicine applications in audiology. Audiology Today, 20, 36-41.

Lancaster P., Krumm M., Ribera J. \& Klich R. 2008. Remote hearing screenings via telehealth in a rural elementary school. Am J Audiol. 17, 114-122.

Laplante-Levesque A., Pichora-Fuller K.M. \& Gagne J-P. 2006. Providing an internet-based audiological counselling programme to new hearing aid users: A qualitative study. Int J Audiol, 45, 697-706.

Lopez A.D., Mathers C.D., Ezzati M., Jamison D.T. \& Murray C.J.L. 2006. Global burden of disease and risk factors. New York: Oxford University Press.

Margolis R.H. \& Morgan D.E. 2008. Automated pure-tone audiometry: an analysis of capacity, need, and benefit. Am J Audiol 17, 109-113.

Margolis R.H., Saly G.L., Le C. \& Laurence J. 2007. Qualind: A method for assessing the accuracy of automated tests. J Am Acad Audiol,18, 78-89.

Mars M. 2008. Telemedicine in KwaZulu-Natal, South Africa: from failure to cautious optimism? J Telemed Telecare, 13(Supp3), 57-59.

Melo T.M. 2008. Educação a distância na capacitação de agentes comunitários de saúde na área de saúde auditiva infantil. Faculdade de Odontologia de Bauru - Universidade de São Paulo. Dissertação (Mestrado). Bauru.

McPherson B. 2008. Audiology: A developing country context. In: B. McPherson \& R. Brouillette (eds.). Audiology in developing countries. Hauppauge, NY: Nova Publishers, pp. 520. 
Mishra S.K., Pradeep P.V. \& Mishra A. 2009. Telementoring in India: Experience with endocrine surgery. In: R. Wootton, N.G. Patil, R.E. Scott \& K. Ho (eds.) Telehealth in the developing world. London: Royal Society of Medicine Press Ltd, pp. 109-120.

Moeller M.P., Tomblin J.B., Yoshinaga-Itano C., Connor C.M., Jerger S. 2007. Current state of knowledge: language and literacy of children with hearing impairment. Ear Hear, 28, 740-753.

Olusanya B.O. 2004. Self-reported outcomes of aural rehabilitation in a developing country. Int J Audiol, 43, 563-571.

Olusanya B.O., Ruben R.J. \& Parving A. 2006. Reducing the burden of communication disorders in the developing world: an opportunity for the millennium development project. JAMA, 296, 441-444.

Olusanya B.O. \& Newton V.E. 2007. Global burden of childhood hearing impairment and disease control priority project for developing countries. Lancet, 369, 1314-1317.

Olusanya B.O., Emokpae A., Renner J.K. \& Wirz S.L. 2009. Costs and performance of early hearing detection programmes in Lagos, Nigeria. Trans R Soc Trop Med Hyg, 103, 179-186.

Olusanya B.O., Swanepoel D., Chapchap M.J., Castillo S., Habib H., Mukari S.Z., Martinez N.V., Lin H.C. \& McPherson B. 2007. Progress towards early detection services for infants with hearing loss in developing countries. BMC Health Serv Res,, 7,14.

Ramos A., Rodriguez C., Martinez-Beneyto P., Perez D., Gault A., Falcon J.C. \& Boyle P. 2009. Use of telemedicine in the remote programming of cochlear implants. Acta Otolaryngol, $129,533-540$. 
Rao B. \& Lombardi A. 2009. Telemedicine: current status in developed and developing countries. J Drugs Dermatol, 8, 371-375.

Ribera J. 2005. Interjudge reliability and validation of telehealth applications of the Hearing in Noise Test. Semin Hear, 26, 13-18.

Schmiedge J. 1997. Distortion product otoacoustic emissions testing using telemedicine technology. Unpublished masters thesis, Minot State University, Minot, North Dakota.

Shapiro W.H., Huang T., Shaw T., Roland J.T. \& Lalwani A.K. 2008. Remote intraoperative monitoring during cochlear implant surgery is feasible and efficient. Otol Neurotol, 29, 495498.

Smith A .C., Dowthwaite S., Agnew J. \& Wootton R. 2008. Concordance between real-time telemedicine assessments and face-to-face. Med J Aust, 188, 457-460.

Smits C., Kapteyn T. \& Houtgast T. 2004. Development and validation of an automatic speechin-noise screening test by telephone. Int J Audiol, 43, 15-28.

Smits C., Merkus P. \& Houtgast T. 2006. How we do it: The Dutch functional hearing screening tests by telephone and internet. Clin Otolaryngol, 31, 436-340.

Swanepoel D. 2008. Infant hearing loss in developing countries-a silent health priority. Audiology Today, 20, 16-24.

Swanepoel D., Störbeck C. \& Friedland P. 2009. Early hearing detection and intervention in South Africa. Int J Pediatr Otorhinolaryngol, 73, 783-786. 
Theunissen M. \& Swanepoel D. 2008. Early hearing detection and intervention services in the public health sector of South Africa. Int J Audiol 47, S23-S29.

Towers A.D., Pisa J., Froelich T.M. \& Krumm M. 2005. The reliability of click-evoked and frequency-specific auditory brainstem response testing using telehealth technology. Semin Hear, 26, 19-25.

Wesendahl T. 2003. Hearing aid fitting: application of telemedicine in audiology. Int Tinnitus J, 9, 56-58.

WHO 2006a. Primary ear and hearing care training manuals. Geneva: World Health Organization. http://www.who.int/pbd/deafness/activities/hearing_care/en/index.html (Accessed 11 June 2009)

WHO 2006b. Deafness and hearing impairment. Geneva: World Health Organization. http://www.who.int/mediacentre/factsheets/fs300/en/index.html (Accessed 15 May 2009)

WHO 2008a. The global burden of disease: 2004 update. Geneva: World Health Organization. http://www.who.int/healthinfo/global_burden_disease/GBD_report_2004update_full.pdf (Accessed 14 May 2009)

WHO 2008b. World Health Statistics 2008. Geneva: World Health Organization. www.who.int/whosis/whostat/EN_WHS08_Full.pdf (Accessed 14 May 2009)

Wootton R., Ho K., Patil N.G. \& Scott R.E. 2009. Introduction. In: R. Wootton, N.G. Patil, R.E. Scott \& K. Ho (eds.) Telehealth in the developing world. London: Royal Society of Medicine Press Ltd, pp. 3-8. 
Wootton R. 2009. The future use of telehealth in the developing world. In: R. Wootton, N.G.

Patil, R.E. Scott \& K. Ho (eds.) Telehealth in the developing world. London: Royal Society of Medicine Press Ltd, pp. 299-308.

Wynchank S. \& Fortuin J, 2008. Telepaediatrics, primary health care and developing countries. South African Journal of Child Health, 2, 158-160.

Yates J.T. \& Campbell K.H. 2005. Audiovestibular education and services via telemedicine technologies. Semin Hear, 26, $35-42$

Yoshinaga-Itano C., Sedey A., Coulter D. \& Mehl A. 1998. Language of early- and lateridentified children with hearing loss. Pediatrics, 102, 1161-1171.

Yoshinaga-Itano C. 2004. Levels of evidence: universal newborn hearing screening (UNHS) and early hearing detection and intervention systems (EHDI). J Commun Disord, 37, 451-465. 